\title{
Patient speaking: Hospice patients discuss their care
}

\author{
Carolyn Pevey, PhD
}

\begin{abstract}
Much research examines the effects of hospice assistance on the primary caregivers of hospice patients, but very little systematic research has investigated the perceptions of hospice patients themselves of the care they receive. This researcher asked 38 hospice patients whether and how hospice provided comfort, allowing the patients to guide discussion of services. Patients reported that hospice helped them by providing human contact, allowing open discussion and communication with other health professionals, providing assistance with tasks they could no longer do for themselves, and providing physical measures to alleviate discomfort.

Key words: hospice, end-of-life care, patient/caregiver relations, communication
\end{abstract}

\section{Introduction}

Although hospices send questionnaires to bereaved loved ones after a death, and registered nurses (RNs) monitor the comfort levels of hospice patients while they're alive, virtually no systematic inquiry exists regarding

Carolyn Pevey, PhD, Auburn University Montgomery, School of Liberal Arts, Montgomery, Alabama. patient satisfaction with hospice services. This paper reports on satisfaction with hospice care for 38 patients who were receiving hospice benefits.

\section{Methods}

\section{Patient selection}

Nursing staff of the central Texas hospice where this research was conducted (Cedar Hospice is a pseudonym) asked appropriate patients whether they would like to participate in this study before the investigator called to set up interview appointments. Appropriateness criteria included physical stability, at least four weeks experience as a hospice patient, and ability to participate in indepth interviews.

\section{Confidentiality}

All the interviewees were promised strict confidentiality not only because Cedar Hospice insisted on it but also to foster a more open attitude and willingness to talk among the subjects. Interviewees were assured of confidentiality in writing via a consent form. In addition, when their responses became hesitant, they were reminded that they could stop the interview if they wished, and that neither Cedar Hospice nor anyone who read the interviews would be able to identify specific patients' comments. Each interviewee was assigned a pseudonym, and the pseudonyms have been used in this report so that the identity of the respondent cannot be determined.

\section{Study questions}

In the context of a larger study examining avenues of comfort for hospice patients, each person was asked a general question about hospice services: "Has hospice been a comfort to you?" They were then asked, "How has it been a comfort?" Rather than reviewing a checklist of possible areas of satisfaction or dissatisfaction, the patient was given the authority to decide on which services he or she would report. As noted later, patients responded with both general comments about hospice services and comments about individual staff members, particularly the nurses.

\section{Sample demographics}

A roughly proportionate percentage of patients (13.2 percent of patients in this sample versus 11 percent, 14 percent, and 12 percent for the years 1997 to 1999 , respectively) were discharged from Cedar hospice services before dying. Although only 
three of the respondents (7.9 percent) were African-American, Cedar Hospice served an average of 11.4 percent African-American patients between 1995 and 1999. Hispanic patients are also underrepresented in this sample; only one of the interviewees (2.6 percent) was Hispanic versus 11.4 percent Hispanic in the Cedar Hospice patient population from 1995 to 1999. All other participants in this sample (89.4 percent) were white non-Hispanic, although the average white non-Hispanic population at Cedars Hospice from 1995 to 1999 was 75.6 percent.

Twenty-two women and 16 men participated in the study. They ranged in age from 32 to 95 with an average age of 66.9 years. Eleven were divorced, 16 were married, three were single, and eight were widowed. Four had AIDS, two had amyotrophic lateral sclerosis (ALS), 21 had cancer, eight had lung disease (excluding cancer), and four had cardiovascular conditions. One patient had lung disease as well as cancer.

The average time elapsed from hospice admission to interview was 116 days, or just less than four months, with a range from 23 days to approximately one year and eight months (605 days). (These estimates were calculated from some precise and some imprecise answers; consequently, when responses were vague-i.e., "I was admitted in 1999"- the midpoint date of that year was used to arrive at an estimate.) Interviews ranged from 45 minutes to five hours, with most lasting about one-and-a-half hours.

The sample discussed in this report was not randomly selected, and responses cannot be generalized to the US hospice population in general nor the Cedar Hospice patient population. Rather, results are a preliminary investigation into the range of possible responses of patients to their care.

\section{Quality assurance}

Cedar Hospice granted the investigator access to willing patients for these interviews in part because she would be asking patients whether they found hospice services helpful. While all hospice staff participate in monitoring the comfort levels of their patients, patients and caregivers are rarely asked whether they are satisfied with the treatment they are receiving from hospice personnel. In fact, unless the patient is using the services of a nursing assistant whose labor is monitored by an $\mathrm{RN}$, it is unlikely that the patient is ever asked quality-assurance questions. Typically, the staff rely on patient or family complaints to identify which consumers are unhappy with their care. Additionally, the bereavement team sends questionnaires to the survivors of deceased hospice patients (the response rate ranges from 26.3 to 48.9 percent.)

This study was conducted outside these official quality-assurance devices in the hope that the investigator could gather more meaningful data through the use of interviews rather than questionnaires and through input from the patients rather than caregivers. Since the participants knew the interviewer was not a hospice employee, and because she made a point of informing them that their names would not be divulged when she presented her findings, the interviewer effect may have been attenuated.

\section{Satisfaction with comfort}

The interview participants initially had such glowing remarks about the comfort they received from hospice services that the investigator was somewhat suspicious they were merely saying what they presumed she would want to hear. She also wondered whether participants might be concerned that expressing dissatisfaction might damage the relationships they had established with hospice personnel and, by extension, might diminish the level of care they received. For this reason, when initial questions were met with positive remarks, each person was reminded that his or her responses were anonymous and would not be attributable to them. They were also instructed that the hospice wanted to do the best possible job of meeting patients' needs, and that honest responses could only help the organization better meet the needs of future patients. In other words, participants were encouraged to report dissatisfaction to the interviewer.

Surprisingly, however, only two of the 38 people interviewed had negative comments, and even those were notably mild. One woman expressed a desire that nurses set up a routine time for visits so she would be better able to plan for them, and another wished that someone would take care of mowing her lawn at home. These patients were asked whether they would like their nurses to know these issues were distressing them so they could be addressed; both said that they did and gave permission for the investigator to notify the nurses. Although only one of these complaints was nursing-related, the nurses in both cases were notified because they were the contact people between the patient-respondent and the interviewer. (The nurse for the first patient reported understanding the problem and worked to regulate the visiting schedule; the nurse for the latter set up an appointment to have the lawn mowed by a volunteer.) When taken in context of the serious nature of the illnesses of the patients interviewed, neither of these complaints seem particularly troublesome.

\section{Patient responses}

Overwhelmingly, the people interviewed for this study found hospice 
immensely comforting. They often answered the question, "Has hospice been a comfort to you?" with an emphatic, "Yes!" For example, when asked how the hospice could help him more, Mr. Robards, a 77-year-old prostate cancer patient with bone metastasis, replied, "You know, the help has been so much more than I expected, than I even knew existed, that I'm so dumbfounded by it and I just-I couldn't think of any way that they could help me more."

Another patient, Mrs. Micaset, who was dying at age 80 of lung cancer, also had glowing remarks: "The people at hospice are always so cheerful when you call them and you need something. They say they'll get right on it, and they do, immediately. They are really an extraordinary group of people."

When asked to elaborate on how the hospice was a comfort, responses fell into the categories of communication, human contact, and physical attention. As evident from the answers above, some of the responses were not specific to any particular service offered; however, when patients mentioned identifiable services, it was often the nursing department that seemed to have made the biggest impression on them.

\section{Communication}

Several of the interview subjects mentioned the communication benefits when asked to explain how hospice had been helpful. Said Wanda, "Seeing Susie every week is great. She's a good nurse, and talking with her helps me understand what's happening to me."

Wanda was not alone in her appreciation of the help that hospice nurses gave in monitoring and giving her information about the progress of her disease. Lynn Sand, for example, a 41-year-old man with AIDS, put it even more succinctly when he said, "They let me know where I stand."
Cameron Green, a 44-year-old AIDS patient, also discussed how helpful his nurse had been. "Last week when I was sick, Susie was here, and she called all these places for me: the doctor, you know, got the pharmacy to bring this IV bag over, and she called me the next day and called me again yesterday to see how I was feeling."

Like Wanda and Lynn, Cameron reported that hospice was most comforting to him because of the communication services his nurse provided, but in his case, it was not communication with him about his disease but communication with the doctor and the pharmacy that he appreciated most. Not only do nurses have easier access to medical office personnel, but having someone else take over such tasks can lift a great burden off very ill people.

Respondents also appreciated the openness and listening that hospice staff provided. For people whose relatives are in denial yet themselves know the truth about the severity of their illness, there is some comfort in having people who are willing to discuss death with them. The now classic insights of Kübler-Ross ${ }^{1,2}$ regarding the need for dying people to communicate with others about their illness were reflected in interview responses during this study.

Betty Specter, a 55-year-old divorced woman with heart disease, clarified what hospice gives her.

Tremendous comfort. They're just the most caring, loving, compassionate group of people I've ever known, and they can talk to me openly about death, and they can use the word "terminal" and look me in the eye and not get all teary-eyed, and I mean, we're able to talk about it and there just are not many people... [most people] avoid the subject like the plague.

Thus, it was not just having someone with whom they could talk but having someone to whom they could talk candidly about dying that was so important to participants. Part of the ability of hospice personnel to communicate well with dying patients is that they can maintain some emotional distance as opposed to family and close friends. Simmel ${ }^{3}$ argued that a stranger has a special position in any community because the stranger is capable of being both involved and objective at the same time. This stranger,

unbound by roots to the particular constituents and partisan dispositions of the group ... confronts [them] with a distinctly objective attitude. [This] attitude ... does not signify ... detachment and nonparticipation; [rather, it] is a distinct structure composed of remoteness and nearness, indifference and involvement.

Hospice personnel may function as this type of stranger, particularly since good hospices train personnel in all departments to allow patients to talk about their conditions.

\section{Human contact}

Interview respondents made frequent reference to the importance of ongoing human contact that hospice afforded them. Many spoke at length about the caring and compassion that they saw manifested in hospice staff. Evelyn Browning, a 67-year-old patient with chronic obstructive pulmonary disease (COPD), emphasized the importance of this contact in her response. "They're intelligent people; they're interesting people. I enjoy their visits. Rosie, Mary, and Linda give me hugs. You know, the body is actually supposed to have seven hugs a day."

For Evelyn, the physical contact hospice staff provided was a source of 
comfort, but having other people around at all was enough for some respondents. Abel White, a 32-yearold male patient with AIDS who was living in an inpatient hospice at the time of the interview, put it this way:

It's a lot better than living alone. I was living alone before. There are people here. There are people around, and there is life happening-even though everyone here is close to death, they are still living. And there is a real power in the last stages of life that I didn't know about.

For the people who participated in this study, contact with others, whether in the environment or through actual physical touch, was an important benefit of hospice care.

\section{Physical attention}

The third frequently reported area of comfort derived from hospice services was physical attention, primarily centered around the care rendered by nursing staff. Happy Forest, a 65-year-old COPD patient, was comforted by staff assurances that she would not suffer. "[Hospice] is a great, great comfort . . they have assured me that I would not go through any pain, and that's the greatest comfort, that's what I'm afraid of. I can't tolerate pain very well."

Ricky Wheeler, a 41-year-old man with AIDS whose severe discomfort had been eased by the skills and knowledge of the hospice staff, put it most succinctly when he described how the hospice staff had provided comfort. "Mainly [by managing] the pain. 'Let's get it, let's get it right.' They deal with the pain very well."

\section{Competence}

The interview subjects also reported that having a skilled person available for consultation was a great source of comfort to them. Louise Frey, a 75-year-old cancer patient who lived in a nursing home, said that she was comforted by the fact that the hospice nurses made sure the doctor's orders were followed. Ariel Lumas, a 63-year-old woman with uterine cancer who lived at home, also found the competence of hospice nurses comforting. "It just feels like there's somebody I can call if something really bad happens, and they know what they're doing."

\section{Practical assistance}

Finally, interviewees mentioned that the hospice staff had provided comfort by doing jobs for them they were unable to do themselves, from skilled tasks such as filling syringes and assisting with bathing, to tasks requiring less skill such as cooking and light cleaning. In these cases, it was not only the nursing staff but also the volunteers and personal-care aides who were found to be helpful and comforting by these patients.

\section{Discussion}

Almost all comments from the hospice patients who participated in this study were positive. It is clear that the dying people who were interviewed appreciated their hospice care on several levels. The function of most nursing care involves physical attention and technical competence, and the respondents reported receiving comfort from their hospice caregivers in both of these areas. Many people fear death because of the pain they assume it will involve, particularly if the death is prolonged, the person is alert, and perhaps especially, if it results from cancer. Knowing that hospice personnel were committed to keeping them pain-free was an important aspect of comfort for the study participants. They also reported that the nurses' ability to facilitate communication with the doctors and other providers was comforting to them, and they sometimes detailed the difficulties they'd had communicating with their providers before having hospice nurses to do that for them.

Although hospice caregivers are not necessarily uniquely qualified to render positive and nurturing human contact to terminally ill people, they are trained and experienced in treating the dying and as such are perhaps less reticent or fearful in their presence than less experienced staff or those in denial. The willingness of hospice workers to allow communication about terminal illness was comforting to many respondents. Additionally, patients reported that hospice workers provided comfort by taking on routine tasks they were no longer able to do for themselves.

\section{Limitations}

This study is limited by the sampling method as well as by the small number of respondents, and as such the results cannot be generalized. The reports of physically stable patients from one not-for-profit hospice in one city do not necessarily represent data that would be obtained from a much larger random sample. Also, it is possible that the overwhelmingly positive responses are an artifact of some methodological problem, such as having had hospice personnel set up the initial contact between patient and interviewer, and further study controlling for such variables is warranted.

In spite of these limitations, however, the reports of these hospice patients are an intriguing preliminary look at patient satisfaction with services, and this topic deserves further study.

\section{Conclusions}

The hospice patients in this study were overwhelmingly satisfied with 
the care they received from their hospice providers, and with two minor exceptions, there were no complaints. This may indicate that Cedar Hospice is extraordinarily proficient at comforting the dying, but it may also indicate that the nurses recommended those hospice patients who were more likely to provide positive remarks. Future research should make every effort to distance the researcher from the hospice staff, for several reasons. First, patients might feel more free to discuss any dissatisfactions if their hospice staff were not involved in the interview arrangements. Second, a larger number of patients might agree to be in a sample pool if they were making the decision for themselves rather than another person, such as their nurse, making that initial decision for them. Third, in addition to increasing the size of the interview pool, this strategy might increase the range of demographic characteristics found among those willing to participate in such research.

The problems obtaining research data from hospice patients are welldocumented by researchers who have attempted it. Davies et al. ${ }^{4}$ in particular discuss the problem of nursing personnel who believe that interviews are intrusive and who are reluctant to assist researchers. Because nurses may be unwilling to recommend that their patients be offered interviews, it might be helpful if patients were informed in advance that researchers approved by the hospice might occasionally wish to speak with them. They could decide on admission whether to sign a consent to have their names and contact information put on a list to which approved researchers would have access.

\section{References}

1. Kübler-Ross E: Living with Death and Dying. New York: Collier, 1981.

2. Kübler-Ross E: Needs of the dying patient. Paper presented at the Gerontology Center of the University of Southern California in Los Angeles (70007), December $10,1971$.

3. Simmel G: On Individuality and Social Forms (Heritage of Sociology Series, Donald N. Levine, ed.) Chicago, IL: University of Chicago Press, 1971, p.145.

4. Davies B, Reimer J, Brown P, et al.: Challenge of conduction research in palliative care. Omega. 1995; 31(4): 263-273.

\section{Call for Papers}

American Journal of Hospice \& Palliative Medicine invites the submission of hospice-related articles in the following areas: research, case studies, literature reviews, policy examination, and opinion \& commentary.

Suggested topics include, but are not limited to, the following:

- Complementary hospice therapies

- Hospice nursing

- International hospice efforts

- Pain management

- Pediatric hospice care

- Spirituality in hospice

For more information, contact:

Editorial Department

American Journal of Hospice \& Palliative Medicine

470 Boston Post Road

Weston, Massachusetts 02493

Tel: $781-899-2702$

Fax: $781-899-4900$

E-mail: hospice@pnpco.com

Web site: www.hospicejournal.com 